\title{
Perspective-Taking and Social Competence in Adults
}

\author{
Katharine A. McGarry ${ }^{1,2}$, Melanie West' ${ }^{1}$, and Kevin F. Hogan ${ }^{1}$
}

'Department of Health and Behavioural Sciences, Newman University, UK

2 Department of Psychology, University of Johannesburg, South Africa.

\section{KEYWORDS} perspective-taking social competence Theory of Mind
ABSTRACT

Theory of mind (ToM) research assumes an idealized ability in adults (Begeer et al., 2010). Links between ToM and social skills are often presupposed and some researchers argue that claims about the relationship between the two are often broad and unjustified (Hughes \& Leekam, 2004; Liddle \& Nettle, 2006). Perspective-taking (PT) has been heavily implicated in social cognition (Ruby \& Decety, 2004) and is commonly placed under the title of ToM (Baron-Cohen, 2000). However, it is suggested that ToM and PT are not the same skills and may in fact be two distinct forms of social cognition (Cutting \& Dunn, 1999). The current study explored the relationship between PT and social skills within a typically developed adult population. Eighty participants completed the Social Skills Inventory (SSI, Riggio \& Carney, 2003) in addition to a computerised PT task. Greater PT ability was associated with greater social ability. Expressivity and control scores were predicted by PT ability, indicating that greater PT ability is predictive of greater control skills and expressivity skills in individuals and vice versa. Greater emotional intelligence has been associated with superior PT ability and higher expressivity in individuals (Goleman, 1995; Schutte et al., 2001). We suggest that emotional intelligence could be contributing to the relationship between expressivity and PT. Additionally, as both control behaviors and PT abilities place a demand on cognitive resources (Richards \& Gross, 2000; Surtees et al., 2016), we argue that the same executive processes are utilized in both abilities.

\section{INTRODUCTION}

Social competence is the ability of an individual to organize their behavior in a way that is consistent with social morals and conventions in a variety of contexts (Bierman, 2004). Social competence is underpinned by a broad range of abilities commonly referred to as social skills (Reichard \& Riggio, 2008) which are typically classed into three categories: expressivity, sensitivity, and control (Riggio \& Carney, 2003). Expressivity refers to an individual's communication capabilities within a social situation, for instance, their ability to express emotional states (Kring et al., 1994; Riggio \& Carney, 2003; Riggio et al., 2003). Individuals who are more expressive are able to express their felt emotional states and transmit their feelings to others within social interactions more successfully than individuals who are deemed as less expressive (Riggio \& Carney, 2003). Furthermore, expressivity is viewed as a skill in initiating interpersonal interactions. Individuals who are more expressive have greater abilities in engaging others in social interactions than less expressive individuals (Riggio, 1992). Sensitivity relates to an individual's competence in reading and interpreting different social situations and their ability to be aware of the appropriateness of their own actions (Riggio \& Carney, 2003). Individuals who are deemed as more sensitive show greater decoding skills within social situations, for example, greater listening skills and better interpretation of the social situation in comparison to less

Corresponding author: Katharine A. McGarry, School of Psychology, Newman University, Genners Lane, Bartley Green B32 3NT, United Kingdom.

Email:k.graham@newman.ac.uk 
sensitive individuals (Riggio, 1992). An individual's skill in regulating a social interaction, for example, guiding the direction and content of communication, is referred to as control (Riggio \& Carney, 2003; Riggio et al., 2003). Individuals who display greater control have greater abilities in controlling the display of particular emotions on demand, for instance, masking felt emotions by appearing stoic (Riggio, 1992; Riggio \& Carney, 2003). Additionally, individuals who are viewed as more controlled demonstrate superior skills in guiding the direction and content of a social interaction in comparison to less controlled individuals (Riggio \& Carney, 2003).

Successful social interaction is dependent upon an individual's understanding that others may hold different perspectives, motivations, and expectations (Pronin et al., 2002). The ability to understand another individual's mental states and the ability to predict behavior based on these mental states is referred to as theory of mind (ToM, Baron-Cohen, 2000). Many authors assume that ToM is the mechanism which facilitates social skills (Liddle \& Nettle, 2006), helping individuals master social situations (Begeer et al., 2010; Keysar et al., 2003).

In typically developing children and preadolescents, relationships between social behavior and ToM ability have been found (Astington \& Jenkins, 1995; Bosacki \& Astington, 1999; Dockett, 1997; Hughes \& Ensor 2006; Lalonde \& Chandler, 1995; Liddle \& Nettle, 2006; Werner \& Cassidy, 1997). In young children, greater ToM ability has been linked to higher peer ratings of likability and popularity (Dockett, 1997) and greater scores on teacher ratings of social-emotional skills (Lalonde \& Chandler, 1995), in addition to being more skilled with aspects of pretend play (Astington \& Jenkins, 1995). In preadolescents, greater role-taking, empathetic sensitivity, and perspective-taking (PT) ability has been associated with higher teacher and peer ratings of social behavior (Bosacki \& Astington, 1999; Ford, 1982; Liddle \& Nettle, 2006; Pellegrini, 1985).

Further links between ToM and social behavior have been made in atypical populations. Literature has suggested that individuals with disorders such as autism spectrum disorder (ASD) and schizophrenia may struggle in social situations due to ToM deficits (Brüne, 2005; Frith, 1992; Kaland et al., 2002; Roeyers et al., 2001; Volkmar et al., 2004). A high number of symptoms associated with schizophrenia are thought to be related to a compromised ToM (Frith, 1992). Numerous studies have supported this stance, with over thirty studies finding that patients with schizophrenia have major difficulties in understanding the mental states of others (see Brüne, 2005). Similarly, adolescents with ASD have been found to perform worse on advanced measures of ToM when making judgements about the existence and importance of other individuals' mental states (Kaland et al., 2002). Furthermore, high-functioning adults with ASD consistently lack accuracy when labelling another individuals' emotional state (Roeyers et al., 2001).

Although links have been found between ToM ability and social competence within child, preadolescent, and atypical populations (Astington \& Jenkins, 1995; Bosacki \& Astington, 1999; Brüne, 2005; Dockett, 1997; Frith, 1992; Hughes \& Ensor 2006; Kaland et al., 2002; Lalonde \& Chandler, 1995; Liddle \& Nettle, 2006; Roeyers et al., 2001; Volkmar et al., 2004; Werner \& Cassidy, 1997), literature surrounding typical adult populations has received little empirical attention (see Apperly et al., 2009) and assumes an idealized ToM ability (Begeer et al., 2010). Researchers within the field argue that the link between ToM and social behavior has been assumed. As a result, claims about the relationship between the two are often broad and unjustified (Hughes \& Leekam, 2004; Liddle \& Nettle, 2006). ToM is a broad concept, which includes a variety of skills and abilities (Harwood \& Farrar, 2006), such as the ability to attribute mental states to others and understanding that others may have different desires, beliefs, intentions, and perspectives (Baron-Cohen, 2000).

In particular, understanding different perspectives or PT has been heavily implicated in social cognition (Ruby \& Decety, 2004), with successful social interaction being dependent upon an individual understanding that others may hold different perspectives (Pronin et al., 2002). Within the field, however, PT is commonly placed under the title of ToM (Baron-Cohen, 2000) as both ToM and PT require an individual to consider differing or conflicting representations held by another (Harwood \& Farrar, 2006). However, PT is the ability to recognize that other individuals may have different points of view (Johnson, 1975), this ability relates to an individual's sensitivity in recognizing another's mental state, for example, their perceptions (Surtees et al., 2012). Perspective-taking requires an individual to understand the causes of differing perspectives whereas ToM builds on this by requiring an individual to understand how these differing perspectives can influence another individual's thoughts or behavior (Harwood \& Farrar, 2006). Although ToM and PT are closely related, authors have suggested that these abilities are not the same skills and may in fact be two distinct forms of social cognition (Cutting \& Dunn, 1999). Suggestions have been made within the literature that ToM research requires a multi-dimensional approach (Bosacki \& Astington, 1999; Chandler, 1987). Researchers have suggested that the contribution of different aspects of ToM, such as person perspective, need to be investigated to further our understanding of ToM as a concept (Bosacki \& Astington, 1999; Chandler, 1987).

The current study therefore aimed to explore the relationship between PT and social skills within a typically developed adult population. Participants completed a computerized level-1 adult PT task as described by Samson et al. (2010). Level-1 PT tasks require an individual to make a judgement on what another individual does or does not see (Flavell et al., 1981) and is associated with controlled processing, suggesting a need for cognitive control (Surtees et al., 2012; Surtees et al., 2016). Participants also completed the Social Skills Inventory (SSI, Riggio \& Carney, 2003) to obtain an overall score of social skills in addition to scores of expressivity, sensitivity, and control. It was hypothesized that greater SSI scores would be associated with greater PT ability (faster RTs on the PT task), as greater ToM ability has been associated with greater social competence within child, preadolescent, and atypical populations (Astington \& Jenkins, 1995; Bosacki \& Astington, 1999; Brüne, 2005; Dockett, 1997; Frith, 1992; Hughes \& Ensor 2006; Kaland et al., 2002; Lalonde \& Chandler, 1995; Liddle \& Nettle, 2006; Roeyers et al., 2001; Volkmar et al., 2004; Werner \& Cassidy, 1997). Hypotheses regarding the relationship between different social skills (expressivity, sensitivity, and control) and PT ability were not formed due to the limited literature in this area. 


\section{METHOD}

\section{Participants}

Eighty individuals who were recruited from a university in the West Midlands, UK and a local community center in Birmingham, UK participated in the current study (52 females, 28 males). Participants had a mean age of 39.6 years (range: $18-96$ years). Permission was granted by the manager of a local community center for the researchers to attend an activity day and to approach potential participants. Once approached, if individuals showed an interest in participating, the purpose of the study was outlined and individuals were given an information sheet to read. After reading the information sheet, individuals had the opportunity to ask questions and were given a consent form to sign if they wanted to participate. Participants were reminded of their right to withdraw at any point and were given a debrief form which contained the contact details for the researcher in case they had any questions following participation.

\section{Materials}

Each participant completed a demographic questionnaire and the SSI (Riggio \& Carney, 2003). The SSI is a 90 item self-report measure which is formed of six subscales measuring expressivity, sensitivity, and control on two dimensions, emotional (nonverbal) and social (verbal). The SSI requires participants to read different statements and use a 5-point Likert-type scale to indicate how much the statement applies to them $(0=$ Not at all like me, $5=$ Exactly like me $)$. Representative statements for each social skill are provided below:

Expressivity: I have been told that I have expressive eyes and I usually take the initiative to introduce myself to strangers.

Sensitivity: I sometimes cry at sad movies and Sometimes I think that I take things other people say to me too personally.

Control: I am easily able to make myself look happy one minute and sad the next and I am usually very good at leading group discussions. For the current study, an overall social score was obtained for each participant by combining the scores for all the subscales. In addition, scores for expressivity, sensitivity, and control were also obtained by combining social and emotional scores for each social skill.

\section{Stimuli and Procedure}

Participants were shown to a quiet area and completed the demographic questionnaire and the SSI. After the questionnaires had been completed, participants took part in a five-minute computerized adult ToM task on a Lenovo G500s laptop with a 15.6 in. screen. Participants received detailed instructions both verbally and on screen regarding the task and were instructed to respond to each trial as quickly and accurately as possible. Practice trials with feedback had to be successfully completed before responses were recorded. The computerized ToM task was an adapted version of an adult PT task created by Samson et al. (2010), as described by McCleery et al. (2011). OpenSesame software (Mathôt et al., 2012) was used for stimulus presentation and data collection. For each trial, a fixation cross was presented for
$750 \mathrm{~ms}$, followed by an auditory stimulus for $1800 \mathrm{~ms}$. A picture was then displayed on screen until a participant response occurred or for a maximum of $2000 \mathrm{~ms}$. The auditory stimulus either asked for the participants perspective "You see N" or for the avatar's perspective "He sees $\mathrm{N}$ ", where "N" referred to a number from 1 to 3 . The picture stimulus was the presentation of an avatar in a room with between one and three discs on the wall. Participants were required to respond on the keyboard to indicate whether or not the auditory stimulus correctly described the picture. There were 96 trials in total: 48 trials where the participant was required to verify their own perspective (self-perspective) and 48 trials where the participant had to verify the avatar's perspective (other-perspective). For each condition, half the trials (24) were consistent with the participant's perspective and half did not match what the participant observed. Trials were presented in a random order.

\section{Data Analysis}

Incorrect responses where participants did not identify the correct perspective for either themselves or the avatar were removed. Averages were then created for the remaining correct responses in the consistent and inconsistent trials for both perspectives per participant. For example, other-inconsistent RT was measured on the speed of accurate responses for all "He sees N" trials where participants were asked to make a judgement based on the avatar's perspective when the perspective did not match their own. Data was analyzed using SPSS v 21, with two-tailed tests.

\section{RESULTS}

Initial correlation and multiple regression analyses were performed to examine the relationship between perspective and consistency of view with the SSI total score (see Table 1). Preliminary analyses were performed to ensure there was no violation of the assumption of normality, linearity, multicollinearity, and homoscedasticity.

A multiple regression model with all four predictors accounted for $28 \%$ of the variance, $F(4,75)=7.609, p<.01$. As shown in Table 1 , self-consistent, $t(79)=-1.65, p=.102$, and self-inconsistent, $t(79)$ $=-1.82, p=.072$, conditions did not significantly contribute to the model. However, other-consistent, $t(79)=-2.14, p<.05$, and otherinconsistent, $t(79)=1.071, p<.05$, conditions were significant predictors. Therefore, SSI total score was only predicted by conditions where participants were required to make a response based on the avatar's perspective. Faster RTs on the other-perspective trials predicted higher SSI total scores.

To further explore the relationship between SSI score and perspective, RTs were averaged together for other-consistent and otherinconsistent conditions to produce a PT response time. A Pearson product-moment correlation coefficient was computed to assess the relationship between SSI total score, control score, expressivity score, sensitivity score, and PT response time. A moderate, negative correlation was found between PT response time and SSI total $(r=-0.336$, $p<.01)$. The analysis also revealed a moderate, negative correlation between PT response time and control score $(r=-0.336, p<.01)$. 


\begin{tabular}{|c|c|c|c|c|}
\hline & $\begin{array}{l}\text { Average } \\
\text { response time } \\
(\mathrm{ms})\end{array}$ & $\begin{array}{c}\text { Correlation } \\
\text { with SSI } \\
\text { Total } \\
\end{array}$ & $t$ & $B$ \\
\hline Self-consistent & 955.32 & $-.443^{\star}$ & -1.65 & -.400 \\
\hline Self-inconsistent & 1067.18 & $-.430^{*}$ & -1.82 & -.496 \\
\hline Other-consistent & 915.46 & $-.358^{\star}$ & $-2.14^{* *}$ & -.628 \\
\hline Other-inconsistent & 1023.08 & $-.305^{* *}$ & $3.02^{* *}$ & 1.071 \\
\hline
\end{tabular}

Note. SSI $=$ Social Skills Inventory

${ }^{*} p<.01,{ }^{* *} p<.05$

Similarly, a moderate, negative correlation was discovered between PT response time and expressivity score $(r=-.305, p<.01)$. However, a significant correlation was not found between PT response time and sensitivity score $(r=-.067, p=.276)$. Overall, faster response times on the computer task were significantly correlated with higher control, expressivity and SSI total scores.

Two simple linear regressions were preformed to test if PT response time significantly predicted expressivity score and control score. Sensitivity score was not included in the analysis as a significant correlation was not found with PT response time. Preliminary analyses were carried out to ensure there was no violation of the assumption of normality, linearity, multicollinearity, and homoscedasticity. Regression statistics are reported in Table 2.

The regressions revealed that PT response time significantly predicted expressivity scores, $F(1,78)=7.979, p<.01$, and control scores, $F(1,78)=9.911, p<.01$. Expressivity score accounted for $8 \%$ of the variance in PT response time and control score accounted for $10 \%$ of variance in PT response time. These findings suggest that faster response times on the PT task predicted higher expressivity and control scores on the SSI.

\section{DISCUSSION}

As hypothesized, greater performance on the SSI was associated with greater PT ability. The hierarchical regression revealed that SSI total score was only predicted by conditions where participants were required to make a response based on the avatar's perspective. Individuals who had faster accurate response times on the other-perspective trials typically had higher SSI total scores. As a higher SSI total score is indicative of greater social skills (Riggio \& Carney, 2003), this finding indicates that greater PT ability is associated with greater social

\begin{tabular}{lccc}
\hline $\begin{array}{l}\text { TABLE 2. } \\
\text { Linear Regressions Exploring Whether Perspective-Taking }\end{array}$ \\
\multicolumn{4}{l}{ Predicts Expressivity Score and Control Score } \\
\hline R2 & $\mathrm{t}$ & $B$ \\
\hline Expressivity & 0.08 & $-2.83^{*}$ & -0.31 \\
Control & 0.10 & $-3.15^{*}$ & -0.34 \\
\hline
\end{tabular}

${ }^{*} p<.01$ ability in typically developed adults. This finding provides support for previous literature within child, preadolescent, and atypical populations which has found greater ToM ability to be associated with greater social competence (Astington \& Jenkins, 1995; Bosacki \& Astington, 1999; Brüne, 2005; Dockett, 1997; Frith, 1992; Hughes \& Ensor 2006; Kaland et al., 2002; Lalonde \& Chandler, 1995; Liddle \& Nettle, 2006; Roeyers et al., 2001; Volkmar et al., 2004; Werner \& Cassidy, 1997).

Further exploration of the relationship between PT ability and social skills revealed that both expressivity and control were significantly correlated with performance on the computerized PT task. This finding indicates that individuals who demonstrated greater PT abilities also have greater skills in engaging others in social discourse, for example, being able to accurately express their felt emotional states and successfully transmitting feelings to others within social interactions. Furthermore, these results also suggest that these individuals will have greater ability in regards to controlling the display of particular emotions on demand and will demonstrate superior skills in guiding the direction and content of a social interaction. A significant relationship was not found between PT ability and sensitivity. This result indicates that PT ability is not related to an individual's understanding of social behaviors. The findings also revealed that expressivity scores and control scores on the SSI were predicted by performance on the PT task, as indicated by response times in typically developed adults. Greater PT ability is suggested to be predictive of greater control in individuals in terms of regulating emotional and nonverbal displays, in addition to superior skills in guiding the direction and content of communication. Reduced PT ability is suggested to be predictive of poorer control of emotional and nonverbal displays, in addition to reduced skills in guiding the direction of social interactions. Furthermore, the results also indicate that individuals who possess greater PT abilities are more skilled in engaging others in social interactions and are able to accurately express felt emotional states more successfully than individuals with lower PT abilities.

The relationship between PT ability and expressivity can be better understood when looking at literature within the emotional intelligence field (Goleman, 1995, 1998; Saarni, 1999; Schutte et al., 2001). Emotional intelligence is an individual's ability to perceive, understand, regulate, and harness emotions adaptively (Mayer \& Salovey, 1997), in addition to effectively communicating their emotions to others (Goleman, 1995). Similarly, a key aspect of expressivity is the ability to express emotional states to others within social situations (Kring et al., 1994; Riggio \& Carney, 2003; Riggio et al., 2003). Emotional intelligence has also been linked to PT (Schutte et al., 2001) as it is also viewed as cognitive bias which influences our cognitive functions and, in turn, our interpretation of events (Goleman, 1995, 1998). Emotional intelligence is viewed as an essential part of social development and is a significant contributor to the quality of interpersonal relationships (Saarni, 1999). Both expressivity and PT are also seen as important elements of social interactions and interpersonal interactions (Pronin et al., 2002; Riggio, 1992). Expressivity is viewed as a skill in initiating interpersonal interactions, as individuals who are more expressive have greater abilities in engaging others in social interactions (Pronin et al., 
2002; Riggio, 1992). Furthermore, successful social interactions are often dependent upon an individual's understanding that others may hold different perspectives (Pronin et al., 2002). Individuals who are viewed as possessing greater emotional intelligence are able to communicate their emotions more effectively than individuals with lower emotional intelligence (Goleman, 1995). In addition, higher scores for emotional intelligence have been linked to greater PT abilities (Schutte et al., 2001). Given that greater emotional intelligence is associated with superior PT skills and higher expressivity, it could be possible that emotional intelligence could be contributing to the relationship between expressivity and PT. Emotional intelligence could potentially assist an individual in expressing themselves in a social situation in addition to helping them see another individual's perspective.

Support for the relationship between PT ability and control can be found within the executive functioning literature (Samson et al., 2010; Surtees et al., 2012; Surtees et al., 2016). Executive functions are defined as higher-order cognitive abilities which enable individuals to control and coordinate behavior, for example, problem solving, emotion regulation, self-control, and attention (Elliott, 2003). This current finding can be understood in terms of shared executive resources which potentially underpin control behaviors and PT. It is possible that the executive resources allocated for PT may also be allocated for control behaviors. The level-1 PT task completed by participants has been shown to place a demand on executive resources and to require cognitive control (Samson et al., 2010; Surtees et al., 2012; Surtees et al., 2016). Similarly, emotional and social control also place a great demand on an individual's cognitive resources (Richards \& Gross, 2000). It can therefore be argued that individuals are using executive resources to aid them in controlling against displaying felt emotions and guiding the direction of social interactions. These same executive processes may then be allocated in the PT task, enabling individuals to make judgments on the avatar's perspective versus their own. We suggest that individuals with greater cognitive control make faster judgements on the PT task and score higher for control on the SSI.

The results of this study provide support for researchers who have argued that the relationship between ToM and social competence is too broad and requires a multidimensional approach (Bosacki \& Astington, 1999; Chandler, 1987; Hughes \& Leekam, 2004; Liddle $\&$ Nettle, 2006). The current study only found significant relationships between control score and expressivity score in relation to PT. However, different results have been found within the empathy field (Riggio et al., 1989). Riggio et al. (1989) found relationships between empathy aspects of the SSI and independent measures of empathy. The authors suggest that empathy should be viewed as a combination of basic social skills. The differing findings of the current study and Riggio et al's (1989) study indicates that different social skills are associated with different dimensions of ToM. The differences in these findings highlight the benefit of applying a multidimensional approach to ToM research but also demonstrate the need for further research within this field. Although the current study provides useful information about the relationship between PT and social competence, it does not provide an overall picture of the relationship between social ability and ToM.
Future research would benefit from exploring the relationship between all dimensions of ToM (conceptual role-taking, empathetic sensitivity, and PT) and social competence. This would enable the field to develop a clearer picture of the complex relationship between ToM and sociability. Additionally, this would add to the developing literature exploring the limitations of ToM in typical adult populations.

Directionality of the relationship between PT ability and social skills could also be considered a limitation of the current study. As previously discussed, many authors assume that ToM is the mechanism which facilitates social skills (Liddle \& Nettle, 2006). This assumption was adopted in the current study, where the predictive ability of PT was explored in relation to social skills. The findings revealed that expressivity and control skills were predicted by performance on the PT task, with support for these findings being found within the executive function and emotional intelligence literature. It is worth noting however, within the executive functioning field, the relationship between ToM and executive functions has been questioned in regards to which emerges first (Pellicano, 2007). As the authors have argued that control skills are underpinned by executive processes, it could be plausible that social skills in the current study may be predicting PT ability. It is therefore important to be mindful that although expressivity and control skills were found to be statistically related to PT in the current study, there is a degree of uncertainty about the direction of the causal relations amongst these variables.

Another limitation of the current study is that only level-1 PT was explored in relation to social competence. Level-1 PT in adults, although requiring cognitive control, has been demonstrated to be an automatic process (Samson et al., 2010). In comparison, level-2 PT involves understanding that the same visual display may give rise to different visual perspectives (Flavell et al., 1981) and is associated with a higher level of controlled processing, suggesting a greater need for cognitive control (Surtees et al., 2012; Surtees et al., 2016). It would be beneficial to explore both level-1 and level-2 PT tasks in relation to sociability in future studies. This would increase our understanding of the relationship between social competence and person perspective in a typically developed adult population.

Other future considerations for this area would be to explore the contributing roles of gender and age in regards to ToM ability and social competence. Sociability has been shown to be sexually dimorphic and females are described by some as socially superior (Connellan et al., 2000; Lutchmaya et al., 2002). Furthermore, gender differences in peer-related social competence and individual differences in ToM have been noted in young children (Walker, 2005) but not explored in an adult population. In addition, research has demonstrated that ToM ability declines with age (Bernstein et al., 2011) but it is unclear within the literature what, if any, impact this decline has upon social competence. Exploration of both these factors could therefore provide researchers with a deeper insight into the complexities of the relationship between sociability and ToM.

In summary, the current study revealed that greater PT ability is associated with greater social ability in typically developed adults. Additionally, expressivity and control were significantly correlated 
with PT ability but sensitivity was not. Furthermore, expressivity and control scores were predicted by PT ability. This indicates that greater PT ability is predictive of greater control skills and expressivity skills in individuals and vice versa. As both control behaviors and PT abilities place a demand on cognitive resources (Richards \& Gross, 2000; Samson et al., 2010; Surtees et al., 2012; Surtees et al., 2016), we argue that the same executive processes are implicated in both abilities. Therefore, an individual's level of cognitive control underpins their behavioral control within a social situation and their ability to judge another individual's perspective. In addition, greater emotional intelligence has been associated with superior PT ability and higher expressivity (Goleman, 1995; Schutte et al., 2001). Therefore, it is possible that emotional intelligence could be contributing to the relationship between expressivity and PT. The current study provides support for a multi-dimensional approach within ToM research (Bosacki \& Astington, 1999; Chandler, 1987), as the results suggest that different social skills may be associated with different dimensions of ToM. Future research would benefit from exploring the relationship between all dimensions of ToM (conceptual role-taking, empathetic sensitivity, and PT) and social competence in addition to exploring cognitive control and the contributing roles of gender and age. This would allow for a greater insight into the complex relationship that exists between ToM and sociability in typically developed adults.

\section{REFERENCES}

Apperly, I. A., Samson, D., \& Humphreys, G. W. (2009). Studies of adults can inform accounts of theory of mind development. Developmental

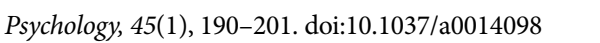

Astington, J. W., \& Jenkins, J. M. (1995). Theory of mind development and social understanding. Cognition \& Emotion, 9(2-3), 151-165.

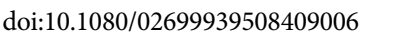

Baron-Cohen, S. (2000) Theory of mind and autism: A fifteen year review. In S.Baron-Cohen, H. Tager-Flusberg, \& D. J. Cohen (Eds.), Understanding other minds. Perspectives from developmental cognitive neuroscience (pp. 3-21). University Press.

Bernstein, D. M., Thornton, W. L., \& Sommerville, J. A. (2011). Theory of mind through the ages: Older and middle-aged adults exhibit more errors than do younger adults on a continuous false belief task. Experimental Aging Research, 37(5), 481-502. doi:10.1080/03 61073x.2011.619466 الس

Begeer, S., Malle, B. F., Nieuwland, M. S. \& Keysar, B. (2010). Using Theory of Mind to represent and take part in social interactions: Comparing individuals with high functioning autism and typically developing controls. European Journal of Developmental Psychology, 7(1), 104-122. doi:10.1080/17405620903024263 山س إس

Bierman, K. L. (2004). Peer rejection: Developmental processes and intervention strategies. Guilford Press.

Bosacki, S., \& Wilde Astington, J. (1999). Theory of mind in preadolescence: Relations between social understanding and social competence. Social Development, 8(2), 237-255. doi:10.1111/14679507.00093 المالسلس

Brüne, M. (2005). “Theory of mind” in schizophrenia: A review of the literature. Schizophrenia Bulletin, 31(1), 21-42. doi:10.1093/schbul/ sbi002 سلس

Chandler, M. (1987). The Othello effect. Human Development, 30(3), 137-159. doi: $10.1159 / 000273174$ 16لس

Connellan, J., Baron-Cohen, S., Wheelwright, S., Batki, A., \& Ahluwalia, J. (2000). Sex differences in human neonatal social perception. Infant Behavior and Development, 23(1), 113-118. doi:10.1016/s0163-6383(00)00032-1 الم

Cutting, A. \& Dunn, J. (1999). Theory of mind, emotion understanding, language, and family background: Individual differences and interrelations. Child Development, 70(4), 853-865.

Dockett, S. (1997, April). Young children's peer popularity and theories of mind [Poster presentation]. The Biennial Meeting of the Society for Research in Child Development, Washington, DC.

Elliott, R. (2003). Executive functions and their disorders Imaging in clinical neuroscience. British Medical Bulletin, 65(1), 49-59. doi:10.1093/bmb/65.1.49 السلسلس

Flavell, J. H., Everett, B. A., Croft, K., \& Flavell, E. R. (1981). Young children's knowledge about visual perception: Further evidence for the Level 1-Level 2 distinction. Developmental Psychology, 17(1), 99-103. doi:10.1037/0012-1649.17.1.99 المبلس

Ford, M. E. (1982). Social cognition and social competence in adolescence. Developmental Psychology, 18(3), 323-340. doi:10.1037/00121649.18.3.323 الس الس

Frith, C. D. (1992). The cognitive neuropsychology of schizophrenia. Psychology Press.

Goleman, D. (1995). Emotional intelligence. Bantam Books, Inc.

Goleman, D. (1998). The emotional intelligence of leaders. Leader to

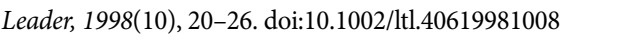

Harwood, M. \& Farrar, M. (2006). Conflicting emotions: The connection between affective perspective taking and theory of mind. British Journal of Developmental Psychology, 24(2), 401-418.

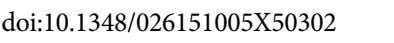

Hughes, C., \& Ensor, R. (2006). Behavioural problems in 2-year-olds: links with individual differences in theory of mind, executive function and harsh parenting. Journal of Child Psychology and Psychiatry, 47(5), 488-497. doi:10.1111/j.14697610.2005.01519.x المالسلس

Hughes, C., \& Leekam, S. (2004). What are the links between theory of mind and social relations? Review, reflections and new directions for studies of typical and atypical development. Social Development, 13(4), 590-619. https://doi/org/10.1111/j.14679507.2004.00285.x السلسلس

Johnson, D. W. (1975). Cooperativeness and social perspective taking. Journal of Personality and Social Personality, 31, 241-244.

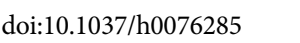

Kaland, N., Møller-Nielsen, A., Callesen, K., Mortensen, E. L., Gottlieb, D., \& Smith, L. (2002). A new 'advanced' test of theory of mind: evidence from children and adolescents with Asperger syndrome. Journal of Child Psychology and Psychiatry, 43(4), 517-528. doi:10.1111/1469-7610.00042 سلس

Keysar, B., Lin, S., \& Barr, D. J. (2003). Limits on theory of mind use in adults.

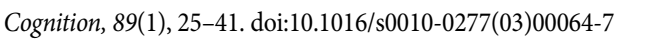

Kring, A. M., Smith, D. A., \& Neale, J. M. (1994). Emotional Expressivity 
Scale (EES) [Database record]. APA PsycTests. doi:10.1037/t01073000 الس

Lalonde, C. E., \& Chandler, M. J. (1995). False belief understanding goes to school: On the social-emotional consequences of coming early or late to a first theory of mind. Cognition \& Emotion, 9(2-3),

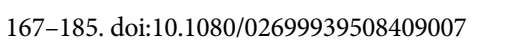

Liddle, B., \& Nettle, D. (2006). Higher-order theory of mind and social competence in school-age children. Journal of Cultural and Evolutionary Psychology, 4(3-4), 231-244. doi:10.1556/ jcep.4.2006.3-4.3 الس الس

Lutchmaya, S., Baron-Cohen, S., \& Raggatt, P. (2002). Foetal testosterone and eye contact in 12-month-old human infants. Infant Behavior and Development, 25(3), 327-335. doi:10.1016/s01636383(02)00094-2 السلسلسل

Mayer, J. D., \& Salovey, P. (1997). What is emotional intelligence? In P. Salovey \& D. J. Sluyter (Eds.), Emotional development and emotional intelligence: Educational implications (pp. 3-34). Harper Collins.

McCleery, J. P., Surtees, A. D., Graham, K. A., Richards, J. E., \& Apperly, I. A. (2011). The neural and cognitive time course of theory of mind. The Journal of Neuroscience, 31(36), 12849-12854. doi:10.1523/ jneurosci.1392-11.2011 الس الس الس

Mathôt, S., Schreij, D., \& Theeuwes, J. (2012). OpenSesame: An open-source, graphical experiment builder for the social sciences. Behavior Research Methods, 44(2), 314-324. doi:10.3758/s13428011-0168-7 سلس

Pellegrini, A. D. (1985). The relations between symbolic play and literate behavior: A review and critique of the empirical literature. Review of Educational Research, 55(1), 107-121. doi:10.3102/00346543055001107 الم

Pellicano, E. (2007). Links between theory of mind and executive function in young children with autism: Clues to developmental primacy. Developmental Psychology, 43(4), 974-990. doi:10.1037/0012-

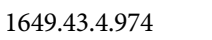

Pronin, E., Puccio, C., \& Ross, L. (2002). Understanding misunderstanding: Social psychological perspectives. Cambridge University Press.

Reichard, R. J., \& Riggio, R. E. (2008). The emotional and social intelligences of effective leadership: An emotional and social skill approach. Journal of Managerial Psychology, 23(2), 169-185. doi:10.1108/02683940810850808

Richards, J. M., \& Gross, J. J. (2000). Emotion regulation and memory: the cognitive costs of keeping one's cool. Journal of Personality and Social

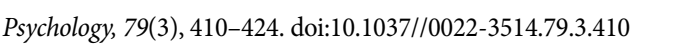

Riggio, R. E. (1992). Social interaction skills and nonverbal behavior. In R. S. Feldman (Ed.), Applications of nonverbal behavioral theories and research (pp. 3-30). Lawrence Erlbaum Associates, Inc.

Riggio, R. E., \& Carney, D. R. (2003). Social Skills Inventory (SSI) [Database record]. APA PsycTests. doi:10.1037/t02651-000 الس الس الس

Riggio, R., Riggio, H., Salinas, C. \& Cole, E. (2003). The role of social and emotional communication skills in leader emergence and effectiveness. Group Dynamics: Theory, Research, and Practice, 7(2), 83-103. doi:10.1037/1089-2699.7.2.83 سلسلسل|

Riggio, R. E., Tucker, J., \& Coffaro, D. (1989). Social skills and empathy. Personality and Individual Differences, 10(1), 93-99.

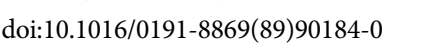

Roeyers, H., Buysse, A., Ponnet, K., \& Pichal, B. (2001). Advancing advanced mind-reading tests: Empathic accuracy in adults with a pervasive developmental disorder. Journal of Child Psychology and

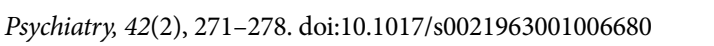

Ruby, P., \& Decety, J. (2004). How would you feel versus how do you think she would feel? A neuroimaging study of perspective-taking with social emotions. Journal of Cognitive Neuroscience, 16(6),

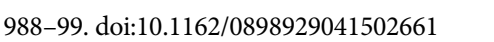

Saarni, C. (1999). The development of emotional competence. The Guildford Press

Samson, D., Apperly, I. A., Braithwaite, J. J., Andrews, B. J., \& Bodley Scott, S. E. (2010). Seeing it their way: Evidence for rapid and involuntary computation of what other people see. Journal of Experimental Psychology: Human Perception and Performance, 36(5), 1255-1266. doi:10.1037/a0018729 السلسلسلس

Schutte, N. S., Malouff, J. M., Bobik, C., Coston, T. D., Greeson C., Jedlicka, C. et al. (2001). Emotional intelligence and interpersonal relations. The Journal of Social Psychology, 141(4), 523-536. doi:10.1080/00224540109600569N Wلس الس الس

Surtees, A. D., Butterfill, S. A., \& Apperly, I. A. (2012). Direct and indirect measures of level-2 perspective-taking in children and adults. British Journal of Developmental Psychology, 30(1), 75-86.

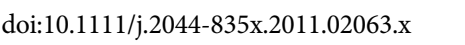

Surtees, A., Samson, D., \& Apperly, I. (2016). Unintentional perspectivetaking calculates whether something is seen, but not how it is seen. Cognition, 148, 97-105. doi:10.1016/j.cognition.2015.12.010 سلسلس

Volkmar, F. R., Lord, C., Bailey, A., Schultz, R. T., \& Klin, A. (2004). Autism and pervasive developmental disorders. Journal of Child Psychology and Psychiatry, 45(1), 135-170. doi:10.1046/j.00219630.2003.00317.x الس الس الس

Walker, S. (2005). Gender differences in the relationship between young children's peer related social competence and individual differences in theory of mind. The Journal of Genetic Psychology,

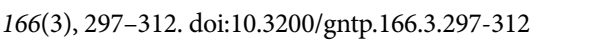

Werner, R., \& Cassidy, K. (1997). Children's psychological understanding and its relationship to social information processing and competence [Poster presentation]. The Biennial Meeting of the Society for Research in Child Development. 